\title{
Features of the solution of a problem of dynamics of a plate with use of the Python programming language
}

\author{
Vladimir Mondrus ${ }^{1,}{ }^{*}$, and Dmitrii Sizov ${ }^{1}$ \\ ${ }^{1}$ Moscow State University of Civil Engineering, 129337, Moscow, Yaroslavskoe shosse, 26, Russia
}

\begin{abstract}
The article describes the features of the application of the modern general-purpose language Python for solving the dynamic problem of oscillations of a rectangular plate. The focus is often on libraries used for numerical computation and visualization of results.
\end{abstract}

\section{Python as the language of numerical calculations}

One of the modern languages of programming of general purpose is the Python language. Feature of writing of programs in this language is limit clarity of a program code, the maximum orientation to increase of labor productivity of the developer [1],[2]. Language is freely extended and has implementations of the interpreter practically for all modern computer platforms.

Considering that fact that the speed of implementation of programs by the interpreter is obviously much lower, than the executed files of the programs written in the compiled programming languages such as Fortran, $\mathrm{C}, \mathrm{C}++$, it is necessary to pay at implementation of computing procedures the main attention to perhaps bigger use of numerical libraries which can be freely used in the Python language. In particular, the free software environment of programming of Anaconda already contains a set of the numerical libraries allowing to possess convenience of manipulation with arrays and array-like objects at the level of the Matlab language, or Fortran in a standard distribution kit, thus the speed of calculations substantially is defined by algorithms of numerical libraries. Using the Python language as the connecting language (language glue) at the appeal to the libraries written in the different compiled languages it is possible to receive the program which is expedited also as well as using the compiled programming languages. It is known that now the community of developers of the Python language at the same time keeps two branches developments of language: Python $2 x$ and Python $3 x$, as reference implementation is most often considered the version of the CPython interpreter (www.python.org/dev/implementations/) though there is also some other interpreters [1],[2], in particular, of Jython, IPython based or on Java-classes, or oriented for work in Microsoft.NET Framework. The special attention is deserved by the experimental version of the PyPy interpreter implemented in the Python language. In scientific calculations it is

*Corresponding author: mondrus@,mail.ru 
most often applied Python2x versions that is explained, first of all by a little more prime object model of language and higher speed of work of Python $2 \mathrm{x}$ in comparison with Python3x.

One of the most often used libraries applied in Python NumPy and SciPy [1] which allow to expand standard objects of Python with arrays, functions for work with arrays, matrix objects, functions of work with polynoms, functions of linear algebra.. Use of these libraries allows to create fast numerical programs so far the majority of operations is made not over scalars, and over arrays and matrixes - data objects of libraries. For display of scientific graphics - matrixes, cuts of arrays the Matplotlib package including the main instruments of display of function graphs of one and many variables is often applied. Use and such means of visualization of results of calculations as Gnuplot possessing the built-in own language and the system of teams is possible.

\section{Modelling of oscillations of a rectangular plate}

The solution of a problem of dynamic calculation of a plate which separate elements have the set external shift is provided in article, sources of vibration influence are on a contour of a plate and in five internal areas in it. Change of coordinates of the points located on a contour of a plate and in it happens under the harmonious law to different frequencies. In Fig. 1 the scheme of a plate taking into account the enclosed shifts is shown.

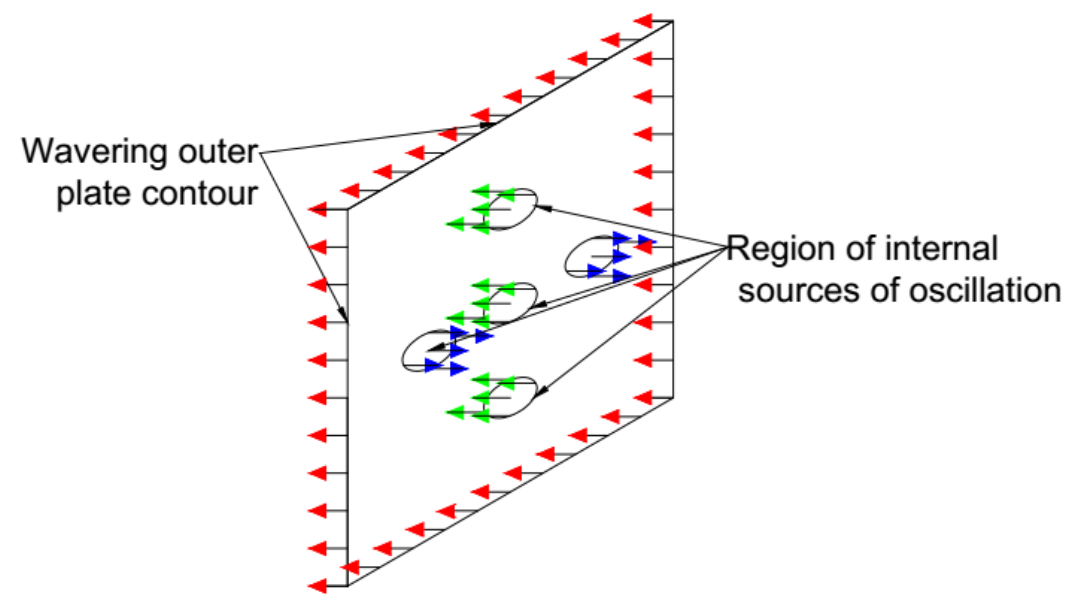

Fig. 1 The arrangement of the sources of oscillations on a square plate.

As appears from [3] at constant flexural rigidity of a plate the equation of fluctuations has an appearance:

$$
D \Delta w+\rho h \frac{\partial^{2} w}{\partial t^{2}}=q(x, t)
$$

where:

$$
D=\frac{E h^{3}}{12 \cdot\left(1-v^{2}\right)}-\text { flexural rigidity of a plate }
$$


$E, \rho, v, h, w$-respectively plate material elastic modulus, density, Poisson's ratio, thickness of a plate and deflection of a median surface.

$q(x, t)$ - external load of a plate, depends on coordinates of a point of application and time $t$.

The equation of fluctuations given in article is guided by the classical theory of KirchhoffLyava. Briefly we will remind its main hypotheses:

1) the rectilinear element, normal to a median surface before deformation remains direct and normal to a median surface, without changing the length;

2) normal stresses between separate layers of a plate $\sigma_{z}=0$

It is convenient to apply the finite difference method based on introduction of a differential grid, splitting area of a plate into separate sites and the further decision of the turned-out algebraic system of equations to the solution of dynamic equation.

As it is known [4],[5] solution of initial boundary value problems for wave equation is possible with use explicit, explicit and implicit and implicit schemes. Use of the explicit scheme allows, being guided by the known solution of a task on the previous time layers at once to determine the decision on the current step by time. This approach does not demand any decision of systems of equations, but imposes rigid restriction on step size on time, at violation of this condition gradual reduction of reliability of the received decision (Courant's condition) is observed. Use of the implicit scheme demands on each of steps of the decision of the turning-out system of equations, as in the sampled equation and unknown, and required sizes on the current temporary step. Explicit and implicit schemes, combine advantages of an explicit method and the guaranteed stability of the implicit scheme. In article for the solution of a task the implicit scheme demanding on each step of the decision of systems of equations, however possessing the proved guaranteed stability of the account is used. In drawings the illustrations characterizing change of a deflection of a plate in different instants are given in Table 1.

Table 1 Field plate deflections at various times

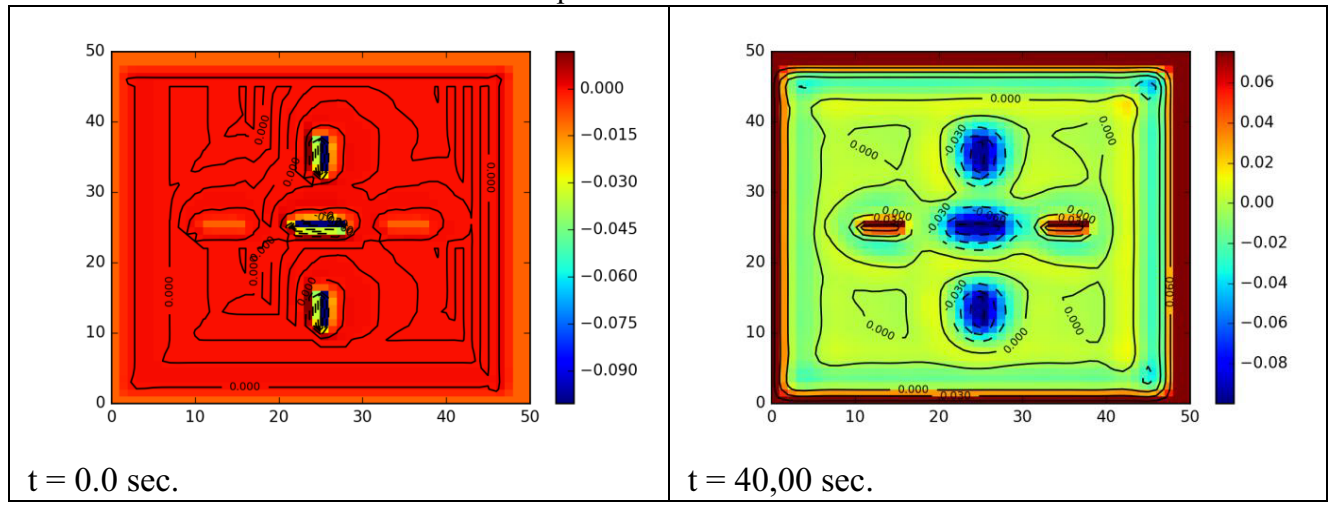




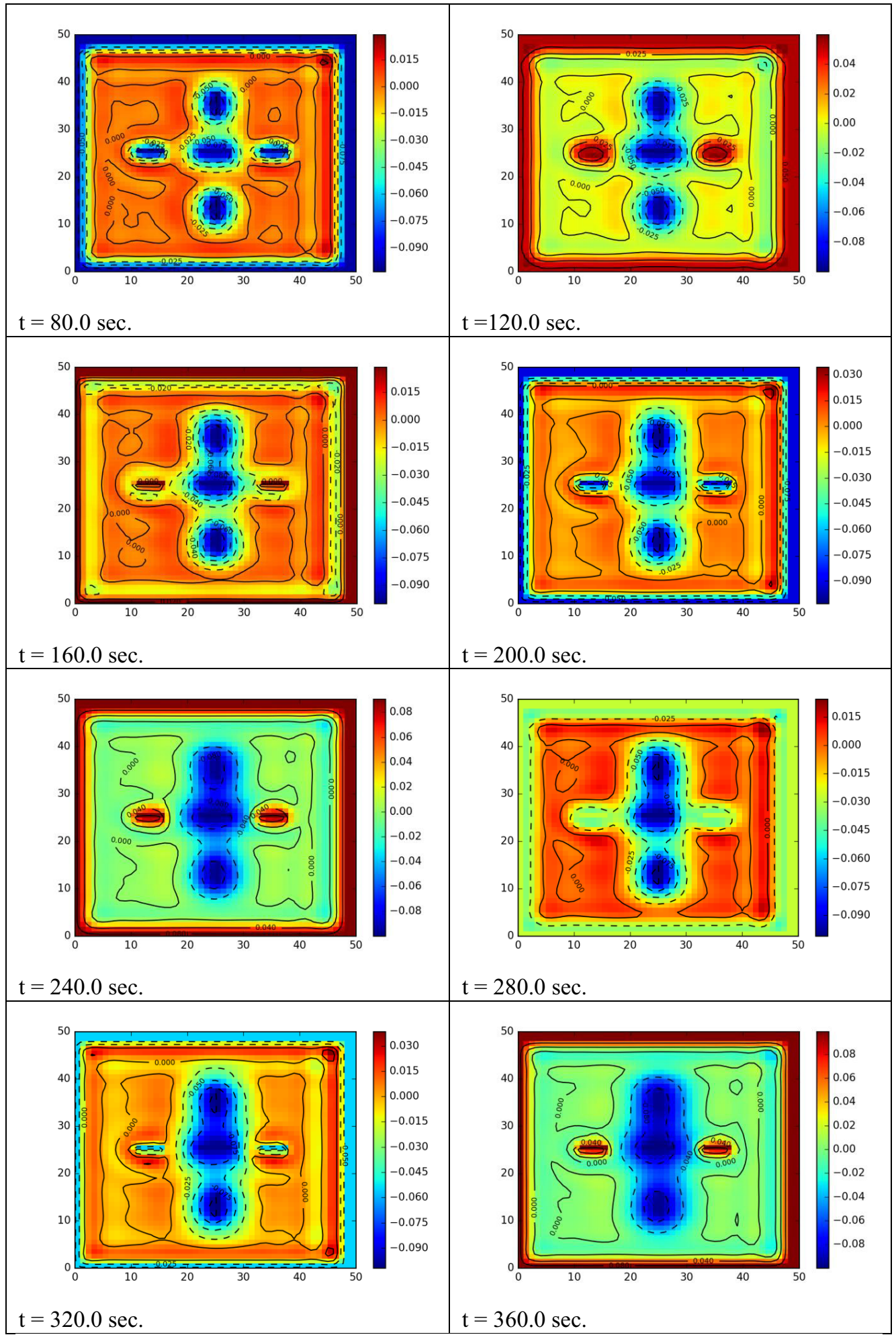

As the conclusion it would be desirable to note that the developed Python-script allows not only to receive the numerical solution of a task for acceptable time, but also in the dynamic 
mode to watch change of the numerical decision on each step of calculation that is reached by use of the connected library of scientific graphics of Matplotlib.

\section{References}

1. Luciano Ramaglio Python To skill tops (M: DMK publishing house, 767 pages ISBN 978-5-97060-384-0, 2016)

2. Wes McKinney Python and the analyses of data ( M: DMK publishing house, 767 pages. ISBN 978-5-97060-315-4, 2015)

3. K. Vasidzu Variation methods in the theory of elasticity and plasticity - (M: World, 542 pages, 1987).

4. E.E. Tyrtyshnikov Numerical analyses: manual for the student. higher education institutions ( M: The publishing center "Akademiya",.320 page ISBN 978-5-76953925-1, 2007)

5. N.P. Savenkova, O. G. Provorova, A.U. Mokin Numerical methods in a mathematical simulation (M: INFA-M ARGAMAK-MEDIA, 176 p, ISBN 978-5-00024-019-9 (ARGAMAK-MEDIA), ISBN 978-5-16-009705-3 (INFA-M, print), 2014) 